\title{
VISÃO DE MÃES SOBRE A HUMANIZAÇÃO NO ATENDIMENTO DA CRIANÇA NA ATENÇÃO PRIMÁRIA À SAÚDE
}

Kálya Yasmine Nunes de Lima1, Akemi Iwata Monteiro², Ana Dulce Batista dos Santos³, Gabriela Bezerra Teixeira ${ }^{4}$

RESUMO: Estudo qualitativo desenvolvido em uma Unidade de Saúde da Família em 2010 com o objetivo de discutir a humanização do atendimento à criança na visão de mães à luz da Política Nacional de Humanização. Os dados foram coletados por meio de formulário aplicado à 12 mães acompanhantes; o enfermeiro foi apontado como o profissional que mais atende a criança. A falta de privacidade, durante o atendimento, as filas de espera, o ambiente inadequado para crianças e responsáveis são fatores que não contribuem para a humanização do atendimento. Há pouco estímulo à participação das mães nas consultas e falhas na comunicação interpessoal entre profissionais e usuários. Para as participantes alguns problemas identificados na Unidade são de fácil resolução tendo por intuito humanizar o atendimento.

DESCRITORES: Humanização da assistência; Saúde da criança; Atenção primária à saúde.

\section{THE VIEW OF MOTHERS ON THE HUMANIZATION OF CARE FOR THE CHILD IN PRIMARY HEALTH CARE}

\begin{abstract}
This qualitative study was undertaken in a Family Health Center in 2010 with the objective of discussing the humanization of the care for the child in the view of the mothers, in the light of the National Humanization Policy. The data was collected through a questionnaire administered to 12 mothers accompanying children; the nurse was indicated as the professional who most cares for the child. The lack of privacy during the attendance, the waiting lines, and the environment which is inappropriate for children and their guardians were factors which do not contribute to the humanization of the attendance. There is little encouragement for the mothers to participate in the consultations, and there are flaws in the interpersonal communication between professionals and service users. For the participants, some problems identified in the Center are easy to resolve, the aim being to humanize the care.
\end{abstract}

DESCRIPTORS: Humanization of care; Children's health; Primary Healthcare.

\section{VISIÓN DE MADRES ACERCA DE LA HUMANIZACIÓN EN EL ATENDIMIENTO DEL NIÑO EN ATENCIÓN PRIMARIA A LA SALUD}

RESUMEN: Estudio cualitativo desarrollado en una Unidad de Salud de la Familia en 2010 con la finalidad de discutir la humanización del atendimiento al niño en la visión de madres a la luz de la Política Nacional de Humanización. Los datos fueron obtenidos por medio de formulario aplicado a 12 madres acompañantes; el enfermero fue indicado como el profesional que más atiende al niño. La falta de privacidad durante el atendimiento, las filas de espera, el ambiente inadecuado para niños y responsables son factores que no contribuyen para la humanización del atendimiento. Hay poco estímulo a la participación de las madres en las consultas y problemas en la comunicación interpersonal entre profesionales y usuarios. Para las participantes, algunos problemas identificados en la Unidad son fáciles de solucionar para humanizar el atendimiento. DESCRIPTORES: Humanización de la asistencia; Salud del niño; Atención primaria a la salud.

\footnotetext{
${ }^{1}$ Enfermeira. Mestranda pelo Programa de Pós-Graduação em Enfermagem da Universidade Federal do Rio Grande do Norte - UFRN. Membro do Grupo de Pesquisa Laboratório de Investigação do Cuidado, Segurança e Tecnologias em Saúde e Enfermagem.

${ }^{2}$ Enfermeira. Doutora em Enfermagem. Professora do Departamento de Enfermagem da UFRN. Membro do Grupo de Pesquisa Cuidado de Enfermagem em Diferentes Fases da Vida.

${ }^{3}$ Enfermeira. Mestre em Enfermagem. Professora do Colegiado de Enfermagem da Universidade Federal do Vale do São Francisco - UNIVASF.

${ }^{4}$ Enfermeira. Residente de Enfermagem em Intensivismo Neonatal pela Residência Integrada Multiprofissional em Saúde da UFRN.
}

Autor correspondente:

Kálya Yasmine Nunes de Lima

Universidade Federal do Rio Grande do Norte

Rua da Piraúna, 106 - 59132-370 - Natal-RN-Brasil

E-mail: lima.yasmine@yahoo.com.br
Recebido: 01/06/2012 Aprovado: 03/06/2013 


\section{INTRODUÇÃO}

Tratar de humanização em saúde implica resgatar o respeito à vida, considerando as circunstâncias sociais, éticas, educacionais e psíquicas do ser humano. Esses valores são incorporados na saúde como complementares aos aspectos técnicos e científicos, que cada vez mais privilegiam a objetividade, generalidade e casualidade no atendimento.

Atendimento humanizado configura-se através da inseparabilidade entre atenção e gestão dos processos de produção de saúde, execução de práticas que aumentam o grau de abertura da comunicação intra e intergrupos e na corresponsabilidade dos sujeitos ${ }^{(1)}$.

No tocante ao atendimento à criança, é necessário refletir sobre a saúde e o desenvolvimento infantil em sua integralidade, compreendendo-o como um processo dependente de diferentes fatores, o qual envolve a família, e não se restringe apenas à ausência de doenças ${ }^{(2-3)}$.

Os princípios norteadores do cuidado à criança abrangem práticas e ações intersetoriais que proporcionam, a cada profissional, o desenvolvimento de ações que contemplem acesso universal, acolhimento, assistência integral, equidade e participação da família. Contribuindo assim para um modelo de assistência que favoreça o acompanhamento e a aproximação das famílias e crianças junto às equipes de saúde na construção de vinculo terapêutico e de atendimento humanizado(4).

$\mathrm{Na}$ atual produção científica muito se tem discutido sobre a humanização na atenção à criança hospitalizada. Porém, a realidade vivenciada mostra que é relevante abordar essa temática nos outros níveis de atenção à saúde, como na atenção primária, principalmente para a área de enfermagem que, notadamente, é a que atua mais próximo dos usuários.

Na prática, o que se observa é que muitos dos princípios supracitados não recebem a devida valorização pelos profissionais e unidades de saúde. Refletindo-se em longo tempo de espera para o atendimento, consultas baseadas apenas em queixas, falta de explicações e esclarecimentos sobre os procedimentos adotados, ausência de espaço na unidade destinada à criança e ambientes pouco acolhedores ${ }^{(2)}$. Configurando-se num atendimento pouco humanizado, que não oferece bem-estar, segurança e conforto para as crianças, pais/ responsáveis e aos próprios profissionais.

Portanto, urge a necessidade de desenvolver ações que estabeleçam uma relação entre os profissionais de saúde e os usuários, em termos de ações humanizadas, tecnicamente competentes, intersetorialmente articuladas e socialmente apropriadas. Mas, para que realmente se obtenha bons resultados diante destas ações, é imperativo o reconhecimento dos profissionais e dos pais/responsáveis sobre o que, de fato, é atendimento humanizado destinado à criança e o que se tem feito para humanizá-lo na atenção primária.

Perante essas implicações, surgiram os seguintes questionamentos: Como as mães avaliam o atendimento da criança na atenção primária à saúde quanto à humanização? O que é realizado, na visão delas, para humanizar o atendimento da criança?

Desta forma, o objetivo deste estudo foi discutir a humanização no atendimento da criança na atenção primária à saúde na visão de mães à luz da Política Nacional de Humanização (PNH).

\section{MÉTODO}

Estudo qualitativo que verifica uma relação dinâmica entre o mundo real e o sujeito, isto é, um vínculo indissociável entre o mundo objetivo e a subjetividade do sujeito que não pode ser traduzido em números ${ }^{(5)}$.

Desenvolvido em uma Unidade de Saúde da Família (USF), localizada no Município de Natal-Rio Grande do Norte, no período de agosto a setembro de 2010. A USF foi elencada para a realização deste estudo por ser o local onde é desenvolvido o projeto de acompanhamento coletivo do crescimento e desenvolvimento da criança, o qual visa construir novas estratégias de atenção à criança, envolvendo a humanização do seu atendimento. A Unidade conta com quatro equipes da estratégia saúde da família, abrangendo em seu total dois médicos, quatro enfermeiras, duas odontólogas, 21 agentes comunitários de saúde, 8 técnicos em enfermagem e duas auxiliares de consultório dentário. Nesta unidade os atendimentos oferecidos às crianças são, em sua maioria, realizados pela equipe de enfermagem, tais como o acompanhamento do crescimento e desenvolvimento da criança, vacinação, triagem neonatal e visita domiciliar. São ainda oferecidos os serviços de odontologia, por meio do Projeto Bebê Sorriso, como também consultas médicas às crianças que apresentam algum problema de saúde de maior gravidade.

A coleta das informações ocorreu mediante entrevista guiada por um formulário semiestruturado que foi preenchido pelos pesquisadores. Este era composto de duas partes: a primeira contendo questões fechadas, que visavam determinar o perfil dos usuários e a segunda com questões abertas que versavam sobre 
como os usuários avaliam o atendimento a criança na USF quanto à humanização e as ações que elegiam como necessárias para melhorar a humanização do atendimento.

Os critérios para inclusão dos participantes no estudo foram: ser mãe ou responsável da criança; estar acompanhando a criança em algum atendimento na unidade na e ser usuária cadastrada na área de abrangência da USF estudada, aceitar e formalizar a participação na pesquisa, mediante assinatura do Termo de Consentimento Livre e Esclarecido. Os critérios de exclusão foram: não possuir vínculo com a criança que está acompanhando para o atendimento (vizinhos ou babás); ser residente de alguma localidade situada fora da área de abrangência da USF que esteja utilizando o serviço de forma esporádica.

Participaram do estudo 12 mães usuárias da USF, que acompanhavam suas crianças nos atendimentos de vacinação, teste do pezinho, acompanhamento do crescimento e desenvolvimento, consultas com o médico e visita domiciliar. O número dos participantes obedeceu ao critério de saturação dos dados ${ }^{(5)}$ e os resultados foram organizados a partir da análise de conteúdo $^{(6)}$ e discutidos à luz da $\mathrm{PNH}^{(7)}$.

Manteve-se o sigilo e anonimato dos participantes denominando-os pela letra $\mathrm{M}$ sequenciada do número correspondente à ordem de entrevistas. $\mathrm{O}$ projeto foi aprovado pelo Comitê de Ética e Pesquisa da Universidade Federal do Rio Grande do Norte sob parecer n. 201/2009, cumprindo as exigências da Resolução 196 do Conselho Nacional de Saúde.

\section{RESULTADOS}

Os participantes da pesquisa possuíam faixa etária entre 19 e 47 anos, sendo todos do sexo feminino e mães acompanhantes. Apenas uma possuía ensino superior completo, duas tinham ensino médio completo, cinco entrevistadas não concluíram o ensino fundamental e quatro possuíam ensino médio incompleto.

A partir de exaustivas leituras sobre os dados obtidos com as mães e da apreciação dos princípios e diretrizes que versam a $\mathrm{PNH}$, surgiram as seguintes categorias analíticas: A formação de vínculo e a corresponsabilização, ambiência e acolhimento e Compreensões sobre a participação social e o protagonismo dos sujeitos.

\section{A formação de vínculo e a co-responsabilização}

Para as entrevistadas, o profissional que oferece mais atendimentos destinados à criança na USF é o enfermeiros. Na visão delas, durante a assistência, enfermeiro e técnicos de enfermagem, bem como os médicos, explicam os procedimentos que estão sendo realizados com a criança e sua importância, porém nem todos os profissionais o fazem, como enuncia a fala:

Existem profissionais que não explicam [...]. Quem explica mais é o enfermeiro. (M4)

[o enfermeiro] Explica sobre os medicamentos, como tomar o sulfato ferroso. (M9)

A explicação clara do que está sendo realizado com a criança foi vista como um fator que humanizaria $o$ atendimento, pois esclarecer os procedimentos, o objetivo e a importância deste estimulam a participação e a autonomia do responsável.

\section{Ambiência e acolhimento}

A porta da sala aberta durante os atendimentos, permitindo o livre acesso de outras pessoas, revela que, segundo as mães, a privacidade durante o atendimento nem sempre é considerada relevante, além das condições econômicas e culturais da criança.

Outro ponto assinalado pela maioria das entrevistadas está relacionado às condições materiais de trabalho. Para elas, o ambiente da USF não é adequado ou acolhedor para uma criança, em decorrência da sujeira, desorganização do ambiente, falta de água para as crianças, carência de medicamentos, ausência de decoração infantil e desconforto durante a espera para o atendimento.

Essas evidências refletem as condições de estrutura física, que apontam a necessidade de distração para as crianças e readaptação da sala de espera como um espaço mais confortável destinado à criança. As mães sugerem:

Colocar televisões e contratar mais profissionais. (M9)

Aumentar a sala de espera e ter uma sala só para crianças. (M2)

\section{Compreensões sobre a participação social e o pro- tagonismo dos sujeitos}

Para as entrevistadas, a participação durante a consulta é estimulada pela maioria dos profissionais, contudo, alguns problemas conjunturais da organização do serviço conduzem à falhas nesse processo de 
estímulo ao protagonismo dos sujeitos. Deste modo, alguns relatos revelam certa insatisfação com o atendimento:

O profissional só estimula a nossa participação quando tem pouca gente prá atender. (M3)

Muitas vezes [o profissional] nem olha direito para a criança. (M8)

Essas atitudes são geradoras de sentimentos de insatisfação por parte dos usuários quanto ao atendimento prestado, criando um distanciamento entre as mães e os profissionais da unidade.

Nesse contexto, foi observado que nenhuma entrevistada apontou se há, ou não, algum estímulo à formação e participação delas em espaços coletivos de gestão que permitam o acordo entre necessidades e interesses de usuários, trabalhadores e gestores.

As ações apontadas pelas mães para melhorar o atendimento à criança quanto à humanização envolveram a facilitação de acesso a exames, maior frequência das visitas domiciliares e, principalmente, a contratação de mais profissionais, como médico pediatra.

\section{DISCUSSÃO}

Promover a criação de vínculo entre os participantes e a produção de sujeitos corresponsáveis diante do processo saúde-doença da criança, considerar os saberes do usuário, além de não centrar as atividades em procedimentos técnicos, buscar refletir e atuar considerando a importância do envolvimento com o usuário, são ações e estratégias que possibilitam aos profissionais firmar uma relação mais próxima com as pessoas que buscam e/ou precisam do serviço ${ }^{(8)}$.

Não obstante, a interação profissional-usuário, através da explanação do que e do porque das ações realizadas, proporciona ao último sentir-se mais à vontade para expor suas dúvidas e anseios. Por isso, a escuta qualificada do usuário e suas demandas permite a criação do vínculo entre profissional e usuário, o que determinará o conhecimento de prioridades de cada um e contribuirá para a resolução dos problemas do usuário ${ }^{(9)}$.

A ambiência e o acolhimento são fatores intrinsecamente ligados ao envolvimento e qualificação dos usuários nos serviços. Deste modo, a falta de privacidade durante os atendimentos reflete que, na realidade dos serviços, as situações do cotidiano se opõem ao princípio de acolhimento, proposto pela PNH. A Política engloba a recepção do paciente, a explicação e privacidade da consulta, como também, a disponibilidade para ouvi-lo, com a finalidade de tornar o usuário confortável para expressar as queixas da criança ${ }^{(7,10)}$.

A problemática do acolhimento e da ambiência não se restringe à falta de locais adequados para as usuárias; persiste a ausência de estratégias por parte dos profissionais para reduzir filas e o tempo de espera. Uma medida sugerida pelas usuárias foi o cumprimento dos horários pelos profissionais. Esse tipo de estratégia pode, além de reduzir o tempo de espera, promover acolhimento, uma vez que proporciona ouvir o usuário e identificar suas necessidades, oferecendo atendimento adequado à demanda ${ }^{(11)}$.

O acolhimento, muitas vezes, é feito de forma errônea, ao se destinar local e profissional específico para essa prática ${ }^{(12)}$. O acolhimento constitui ação inerente à prática de todos os profissionais e em qualquer local ou circunstância dentro do serviço. É pertinente a existência de um ambiente calmo, limpo e confortável que contribua para que o usuário sinta-se acolhido. Isso, desde que essas condições ocorram simultaneamente, como salienta o princípio norteador da $\mathrm{PNH}^{(7)}$ que valoriza a ambiência, com organização de espaços de trabalho saudáveis e acolhedores.

É importante destacar que, alguns problemas apontados pelas entrevistadas não são cabíveis de resolução apenas com a ação dos profissionais da unidade. É necessário esclarecer que a instituição faz parte do Sistema Único de Saúde e deve receber investimentos governamentais para o seu funcionamento adequado e reestruturação, o que por vezes não ocorre, refletindo-se em unidades sucateadas e sem os materiais necessários para realização de seus atendimentos.

Contudo, os profissionais da USF também precisam exercer, além da sua prática cotidiana, a função de promover a criação de ambientes saudáveis, na medida do possível, para estimular a procura do serviço com resultados mais gratificantes tanto para os profissionais quanto para os usuários ${ }^{(13)}$. E isso pode ser feito através de medidas simples, como limpeza e organização do ambiente, evitando que os usuários permaneçam por várias horas em locais desconfortáveis, dentre outros.

A literatura mostra que há diversidade de entendimentos sobre o que é acolhimento, que vai desde a escuta ativa e qualificada até a coleta coleta de exames e opiniões sobre o tempo de espera na unidade, a organização de filas e convocação para atendimento ${ }^{(14)}$. Sob outra ótica, o acolhimento foi apontado apenas como 
o ato de atender a criança, valorizando as atividades curativas e as consultas médicas em detrimento do que seria de fato um atendimento acolhedor.

Partindo-se do princípio que os problemas dos usuários, na atenção primária à saúde, não se encaixam apenas em diagnóstico biomédico, é necessário abordagem ampla, que inclua o reconhecimento de necessidades sociais, sanitárias e familiares que interferem na saúde dos usuários e que propiciem a construção de processo terapêutico individualizado para aquela situação específica ${ }^{(7,15)}$.

Em alguns casos, a assistência prestada ainda não incorpora o princípio norteador da $\mathrm{PNH}$, que defende a construção da autonomia e protagonismo dos sujeitos. Refletindo a persistência de práticas tradicionais nas quais os usuários ainda ocupam posição passiva com pouca participação na tomada de decisões durante a consulta e gestão dos serviços ${ }^{(16)}$.

Contudo, tendo em vista que os indicadores de saúde da criança de uma dada comunidade refletem o estado de toda a população e que a criança depende do cuidado de outros indivíduos para ter um desenvolvimento saudável, faz-se necessário a existência de atitudes que proporcionem às famílias o aumento de autonomia para cuidar dos seus filhos ${ }^{(17)}$.

Essas atitudes podem ser estimuladas na Unidade como também fora dela, através de ações intersetoriais, as quais podem envolver equipamentos sociais, com a finalidade de estabelecer o diálogo com outros setores, operacionalizar parcerias e articular saberes e experiências para a solução dos problemas encontrados. Incentivando, deste modo, a participação da comunidade, uma vez que qualquer mudança na gestão e atenção é mais concreta se erguida com a ampliação da autonomia e vontade das pessoas envolvidas que compartilham responsabilidades.

Por outro lado, fica evidente a manutenção da busca pela assistência curativa centrada na doença. Para os usuários, as atividades voltadas para ações de promoção à saúde e prevenção de doenças são importantes, mas eles ainda não consideram como essenciais para a ida até a unidade de saúde ${ }^{(9)}$. O que é compreensível, tendo em vista ser esse o modelo hegemônico ao longo dos anos.

E é na perspectiva de superar esse modelo que a enfermagem vem atuando, com foco nas ações de promoção à saúde e prevenção de agravos em conjunto com a população, por meio do desenvolvimento de estratégias como a que vem sendo realizada no serviço onde aconteceu o estudo, no intuito de estimular o diálogo e a troca de saberes entre os sujeitos. Logo, almeja-se que a população, ao estar integrada e participando das decisões sobre sua saúde, reconheça a importância dessas atividades e busque a melhoria da saúde, ao invés, apenas, da cura de doenças.

\section{CONCLUSÃo}

Na visão das mães, a humanização no atendimento à criança envolve a formação de vínculo entre profissionais e usuários, o estímulo à participação dos pais/ cuidadores na saúde da criança, o acolhimento e a disposição de espaços com condições físicas e de pessoal adequadas ao atendimento da criança. Contudo, para algumas, o entendimento sobre humanização ainda se encontra-se restrito ao ato de receber atendimento no serviço de saúde.

Foi possível identificar, segundo estas usuárias, uma diferença de atitude entre os profissionais da USF em relação a comunicação, pelo fato de nem todos acolherem e explicarem o atendimento de forma clara aos usuários. Foi relatado também que há muito que melhorar acerca do estímulo ao protagonismo dos sujeitos nas consultas e da participação social nos processos de gestão. Essa participação pode tornar os usuários corresponsáveis e participantes ativos na saúde de suas crianças, além de proporcionar uma relação de confiança entre os profissionais e os usuários.

Para as entrevistadas, alguns problemas encontrados dentro dos espaços da USF são de fácil resolução no intuito de humanizar o atendimento, como a manutenção da privacidade, a redução das filas de espera e alguns aspectos do ambiente. É importante ressaltar que a superação de muitos entraves para a humanização do serviço, principalmente àqueles relacionados à infraestrutura, dependem também de investimentos governamentais. Nesse contexto, ganham importância os espaços coletivos de gestão que reúnem usuários, trabalhadores e gestores para discussão e resolução dos problemas encontrados nas instituições de saúde.

No que concerne ao profissional enfermeiro, o qual muitas vezes é o responsável por coordenar as equipes das USF, e o que mais oferece atendimentos destinados ao público infantil, de acordo com as mães, faz-se necessário reinventar o processo de trabalho de sua equipe, de modo que cada trabalhador seja estimulado a se tornar um agente ativo na elaboração e na prática das mudanças no serviço. Além de incluir os usuários e suas redes sócio-familiares nos processos de cuidado. 


\section{REFERÊNCIAS}

1. Ministério da Saúde (BR). Secretaria-Executiva. Núcleo Técnico da Política Nacional de Humanização. HumanizaSUS. Brasília; 2004.

2. Monteiro AI, Lima KYN, Santos ADB, Teixeira GB, Macêdo IP. Humanização do atendimento à criança na atenção básica: visão dos profissionais. Rev. Rene. 2012;13(4):724-33.

3. Pina JC, Mello DF, Mishima SM, Lunardelo SR. Contribuições da estratégia Atenção Integrada às Doenças Prevalentes na Infância ao acolhimento de crianças menores de cinco anos. Acta Paul. Enferm. 2009;22(2):142-8.

4. Ministério da Saúde(BR). Secretaria de Atenção a Saúde. Agenda de Compromissos para a Saúde Integral a Criança e Redução da Mortalidade Infantil. Brasília; 2004.

5. Minayo MCS. O desafio do conhecimento: pesquisa qualitativa em saúde. São Paulo: Hucitec; 2006.

6. Bardin L. Análise de Conteúdo. Lisboa: Edições 70; 2004.

7. Ministério da Saúde (BR). Secretaria de Atenção à Saúde. Política Nacional de Humanização da Atenção e Gestão do SUS. O HumanizaSUS na atenção básica. Brasília; 2009.

8. Pinafo E, Lima JVC, Baduy RS. Acolhimento: concepção dos auxiliares de enfermagem e percepção de usuários em uma unidade de saúde da família. Rev Espaço para a Saúde. [Internet] 2008;9(2) [acesso em 25 out 2010]. Disponível: http://www.ccs.uel. br/espacoparasaude/v9n2/Artigo\%2053-2007\%20_ Editado_.pdf

9. Franco ECD. A estratégia de saúde da família na perspectiva do usuário. REUFSM. [Internet] 2012;2(1):49-58 [acesso em 02 fev 2012]. Disponível: http://cascavel.ufsm.br/revistas/ojs2.2.2/index.php/ reufsm/article/view/4002/3142

10. Rivemales MCC, Santos PR, Souza PO, Cerqueira RD. Sheltering: a new strategy to humanize the attendance at the health basic attention service Rev. enferm UFPE on line. [Internet] 2009;3(2):371-6 [acesso em 01 jun 2012]. Disponível: http:/www.revista.ufpe.br/revistaenfermagem/ index.php/revista/article/view/304/pdf_879

11. Scholze AS, Ávila LH, Silva MM, Dacoreggio STK. A implantação do acolhimento no processo de trabalho de equipes de saúde da família. Rev Espaço para a Saúde. [Internet] 2006;8(1):7-12 [acesso em 20 set 2010]. Disponível: http://www.ccs.uel.br/espacoparasaude/ v8n1/v8n1_artigo_2.pdf

12. Simões ALA, Rodrigues FR, Tavares DMS, Rodrigues LR. Humanização na saúde: enfoque na atenção primária. Texto Contexto Enferm. 2007;16(3):439-44.

13. Rumor PCF, Bems I, Heidemann ITSB, Mattos LHL, Wosny AM. A promoção da saúde nas práticas educativas da saúde da família. Cogitare enferm. 2010;15(4):674-80.

14. Souza ECF, Vilar RLA, Rocha NSPS, Uchoa AC, Rocha PM. Acesso e acolhimento na atenção básica: uma análise da percepção dos usuários e profissionais de saúde. Cad. Saúde Pública. 2008;24 (1):100-10.

15. Mandú ENT, Gaíva MA, Silva AMN. Necessidades de vida de famílias: desafios para a estratégia saúde da família. Cienc. cuid. saude. 2009;8(4):548-55.

16. Faquinello P, Higarashi IH, Marcon SS. O Atendimento humanizado em unidade pediátrica: percepção do acompanhante da criança hospitalizada. Texto Contexto Enferm. 2007;16(4):609-16.

17. Ferreira JC, Fernandes APP, Souza C, Bicudo DO, Mazza VA. A percepção do gestor sobre a organização da atenção básica à saúde da criança. Cogitare enferm. 2010;15(1):26-32. 